\title{
Stereoselective Synthesis of the C19-C39 Fragment of Bastimolide A
}

\author{
Nemilikonda Sravan Kumar ${ }^{a, b}$ \\ B. Janaki Ramulua \\ Subhash Ghosh*a,b iD \\ ${ }^{a}$ Department of Organic Synthesis \& Process Chemistry, CSIR-Indian \\ Institute of Chemical Technology, Hyderabad-500007, India \\ subhash@iict.res.in \\ b Academy of Scientific and Innovative Research (AcSIR), Ghaziabad \\ 201 002, India
}

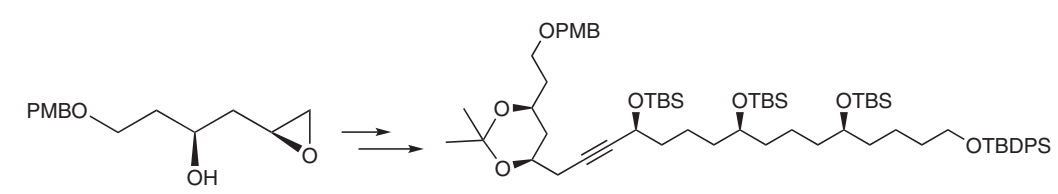

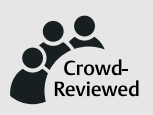

Accepted after revision: 16.09.2021

Published online: 17.09 .2021

DOI: 10.1055/a-1647-7202; Art ID: so-2021-d0043-op

License terms: $(c)(i)=($

(C) 2021. The Author(s). This is an open access article published by Thieme under the terms of the Creative Commons Attribution-NonDerivative-NonCommercial-License, permitting copying and reproduction so long as the original work is given appropriate credit. Contents may not be used for commercial purposes or adapted, remixed, transformed or built upon. (https://creativecommons.org/licenses/by-nc-nd/4.0/)

Abstract This paper describes the synthesis of the C19-C39 fragment of the antimalarial natural product bastimolide $A$ via addition of a functionalized C19-C26 alkyne fragment to a C27-C39 aldehyde fragment. Opening of a terminal epoxide and Noyori asymmetric reduction were used as key steps in the synthesis.

Key words bastimolide A, antimalarial activity, stereoselective synthesis, epoxide opening, Noyori asymmetric reduction

Large numbers of novel bioactive secondary metabolites have been isolated from marine organisms and many of them are under clinical development against various diseases. ${ }^{1}$ Bastimolide A (1, Figure 1$)$, a polyhydroxylated macrolide, was isolated by Gerwick and co-workers from the cyanobacterium Okeaniahirsuta collected from the Caribbean coast of Panama. ${ }^{2}$ Detailed NMR studies, followed by singlecrystal X-ray diffraction study of a nona-p-nitrobenzoate derivative confirmed its structure. Bastimolide A has shown antimalarial activity against four resistant strains of $P$. falciparum with $\mathrm{IC}_{50}$ values between 80 and $270 \mathrm{nM}$. Its attractive architecture along with potent antimalarial activity and our interest in the total synthesis of complex natural products $^{3}$ drew our attention to attempt its total synthesis. So far only one synthetic study has been reported by Quintard et al. where the synthesis of the C15-C27 fragment of bastimolide A was achieved by implementing enantioselective catalytic reactions. ${ }^{4}$ In this communication, we report the synthesis of a key C19-C39 fragment of bastimolide A.

Retrosynthetically, bastimolide A could be synthesized (Scheme 1) from aldehyde $\mathbf{2}$ that would be obtained from $\mathbf{3}$ via deprotection of the PMB ether, followed by oxidation of

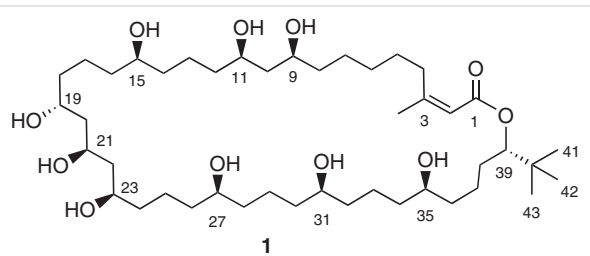

Figure 1 Bastimolide A (1)

the resulting alcohol. Fragment $\mathbf{3}$ might be obtained through coupling of alkyne $\mathbf{5}$ and aldehyde $\mathbf{4}$.

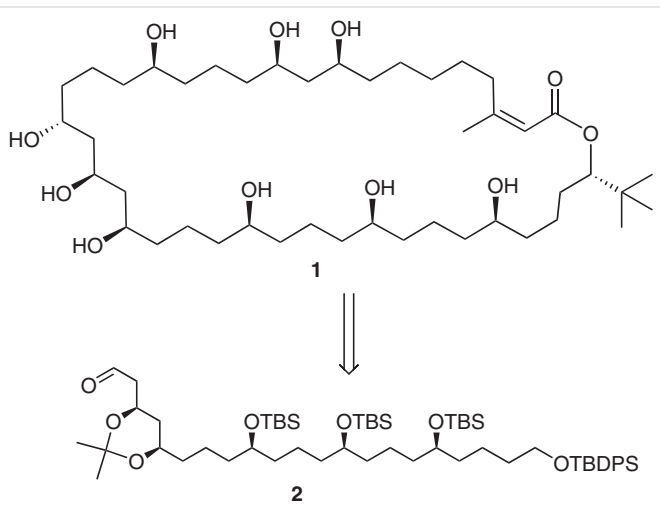

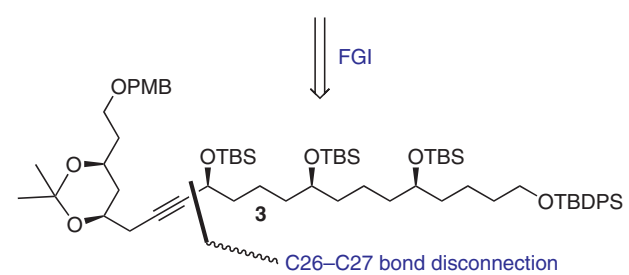<smiles>C#CCC1CC(CCOC)OC(C)(C)O1</smiles>

Scheme 1 Retrosynthetic analysis 
Following this retrosynthetic plan, the synthesis of $\mathbf{5}$ was commenced (Scheme 2) from known epoxy alcohol $\mathbf{6}$, which was prepared from 3-[(4-methoxybenzyl)oxy]propan-1-ol according to the reported procedure. ${ }^{5}$ TBS protection of $\mathbf{6}$ under standard conditions afforded $\mathbf{7}$ in low yield (30\%). However TBS protection in presence of $\mathrm{AgNO}_{3}$ gave 7 in good yield. ${ }^{6}$ Epoxide 7 was then opened with lithium trimethylsilylacetylide ${ }^{6}$ in the presence of $\mathrm{BF}_{3} \cdot \mathrm{Et}_{2} \mathrm{O}$ to give homopropargylic alcohol 8. Both the silyl protecting groups were removed with $\mathrm{TBAF}^{7}$ to give diol $\mathbf{9}$ that, on acetonide protection, ${ }^{8}$ furnished the alkyne fragment $\mathbf{5}$.

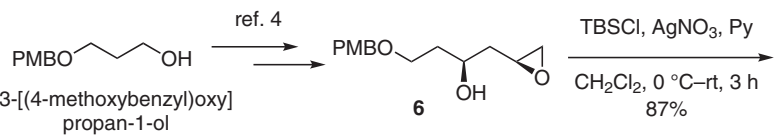

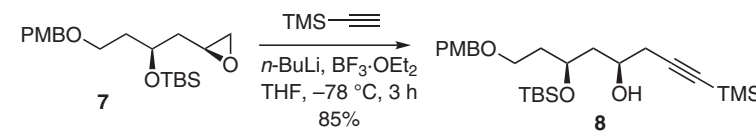

$$
\begin{aligned}
& \underset{\substack{0 \\
{ }^{\circ} \mathrm{C}-\mathrm{rt}, 1 \mathrm{~h}}}{\stackrel{\mathrm{h}}{\mathrm{TBAF}} \text { PMBO }}
\end{aligned}
$$

Scheme 2 Synthesis of compound $\mathbf{5}$

The synthesis of aldehyde fragment $\mathbf{4}$ is depicted in Scheme 3. Opening of known epoxide $\mathbf{1 0}^{9}$ with the anion of trimethylsilylacetylene ${ }^{10}$ gave compound $\mathbf{1 1}$ that on TMS deprotection followed by TBS protection of $\mathbf{1 2}$ gave compound $\mathbf{1 3}$ in $91 \%$ yield. Addition of alkyne $\mathbf{1 3}$ to the known aldehyde $\mathbf{2 0}^{11}$ gave a diastereomeric mixture of alcohol $\mathbf{1 4}$ (1:1) that on DMP ${ }^{12}$ oxidation followed by asymmetric reduction of the resulting ketone $\mathbf{1 5}$ under Noyori conditions ${ }^{13}$ furnished diastereomerically pure compound $\mathbf{1 6}(\mathrm{dr}$ 97:3). TBS protection of the alcohol $\mathbf{1 6}$ followed by one-pot benzyl deprotection and reduction of the alkyne functionality gave primary alcohol $\mathbf{1 8}$ that on DMP oxidation furnished the aldehyde fragment 4.

Having both the fragments $\mathbf{4}$ and $\mathbf{5}$ in hand, we planned their coupling (Scheme 4). Accordingly, alkyne $\mathbf{5}$ on treatment with $n$-BuLi followed by reaction of the resulting anion with the aldehyde $\mathbf{4}$ furnished alcohol 21, oxidation of which with DMP afforded the corresponding ynone. Asymmetric reduction of this under Noyori conditions, followed by TBS protection of the resulting alcohol, afforded the pure C19-C39 fragment 3 of bastimolide A after chromatography.

In summary, a convergent approach for a convenient synthesis of C19-C39 fragment of bastimolide A has been achieved via addition of alkyne $\mathbf{5}$ to an aldehyde $\mathbf{4}$. The synthesis was completed in 17 steps from known compounds $\mathbf{1 0}$ and $\mathbf{6}$ with an overall yield of $6.9 \%$. Key reactions used in
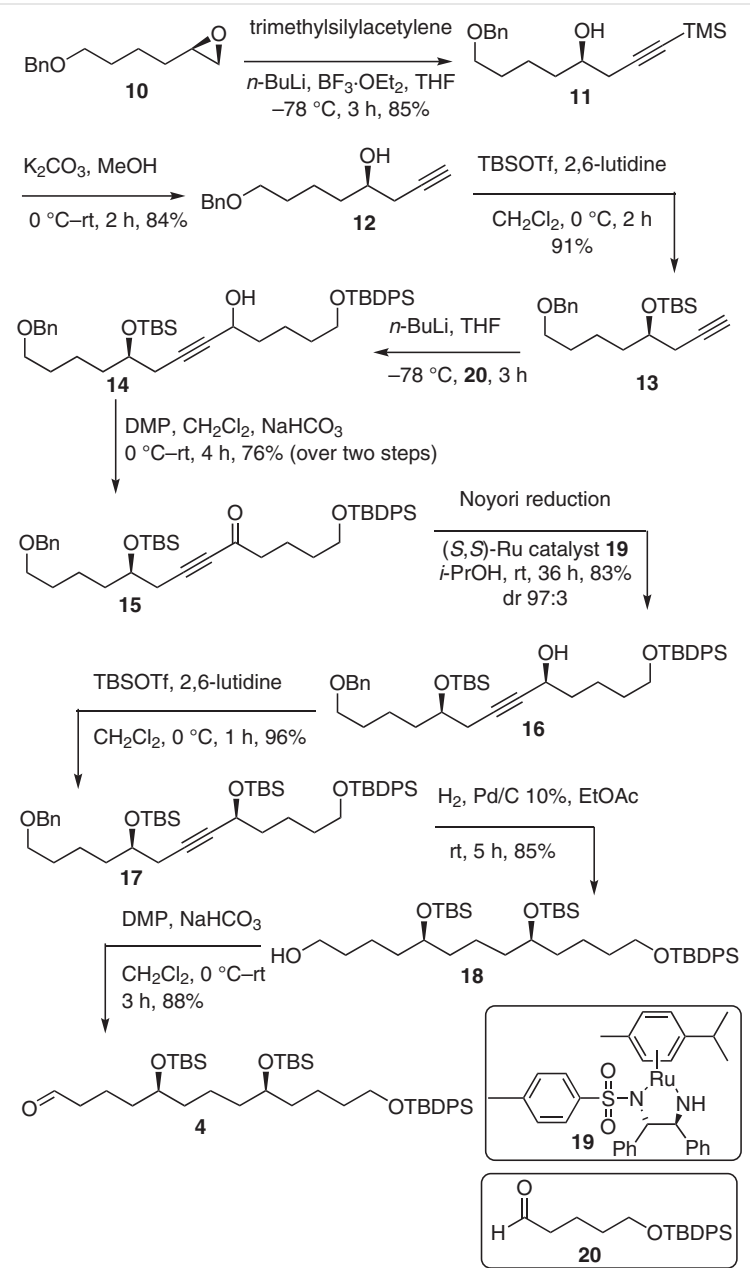

Scheme 3 Synthesis of compound 4
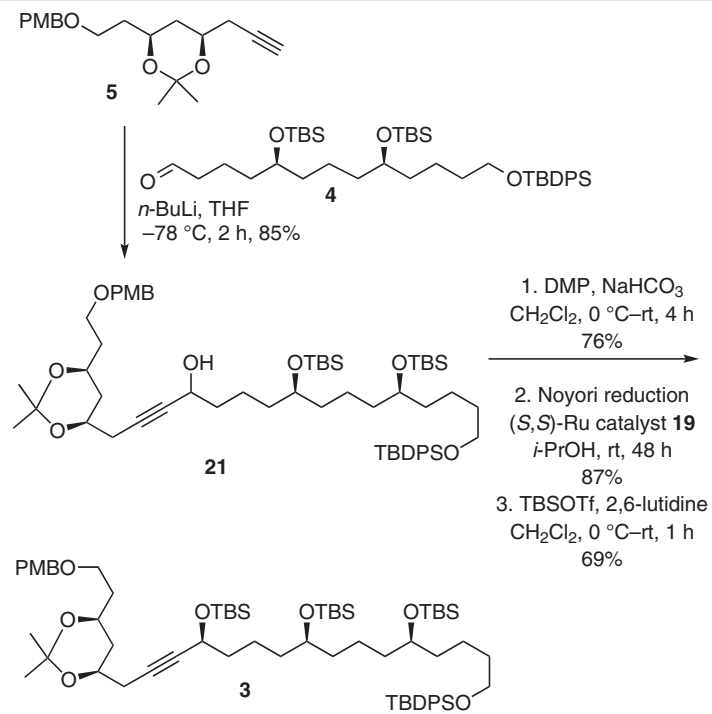

Scheme 4 Synthesis of the C19-C39 fragment of bastimolide A 
the synthesis include opening of a terminal epoxide with an alkyne anion and asymmetric reduction of the ynone. Furthermore, the chemistry reported here can be used for the large-scale preparation of $\mathbf{3}$.

All the starting materials, reagents, and solvents were used as received without further purification, unless otherwise stated. Reactions were monitored by thin-layer chromatography (TLC) carried out on silica-coated plates and components were visualized with UV light. ${ }^{1} \mathrm{H}$ NMR and ${ }^{13} \mathrm{C}$ NMR spectra were recorded at 500 and 400 $\mathrm{MHz}$ on Bruker spectrometers in either $\mathrm{CDCl}_{3}$ or DMSO- $d_{6}$ with TMS as an internal standard. IR spectra were obtained on a Bruker Alpha spectrophotometer (Opus 8.2). Mass spectra were obtained on an AB SCIEX QTrap 5500 LCMS/MS system. The mass spectra (HRMS) were recorded using either a TOF or double focusing spectrometer.

\section{tert-Butyl $(\{(S)-4-[(4-M e t h o x y b e n z y l) o x y]-1-[(S)-o x i r a n-2-y 1]-$ butan-2- yl\}oxy)dimethyl Silane (7)}

To a stirred solution of compound $\mathbf{6}(5.0 \mathrm{~g}, 19.8 \mathrm{mmol})$ in anhydrous $\mathrm{CH}_{2} \mathrm{Cl}_{2}(100 \mathrm{~mL})$ were added sequentially TBSCl $(5.97 \mathrm{~g}, 39 \mathrm{mmol})$, $\mathrm{AgNO}_{3}(6.74 \mathrm{~g}, 39 \mathrm{mmol})$, and pyridine $(1.75 \mathrm{~mL}, 21 \mathrm{mmol})$ at $0{ }^{\circ} \mathrm{C}$ under nitrogen, and the mixture was stirred for $1 \mathrm{~h}$ at $0{ }^{\circ} \mathrm{C}$ and $2 \mathrm{~h}$ at rt. The reaction mixture was quenched with aq $\mathrm{NaHCO}_{3}(60 \mathrm{~mL})$ and extracted with EtOAc $(2 \times 200 \mathrm{~mL})$. The combined organic layers were washed with $\mathrm{H}_{2} \mathrm{O}(100 \mathrm{~mL})$ and brine $(50 \mathrm{~mL})$, dried over $\mathrm{Na}_{2} \mathrm{SO}_{4}$, filtered, and concentrated under vacuum. Purification of the crude product by column chromatography $\left(\mathrm{SiO}_{2}, 15 \%\right.$ EtOAc in hexane) afforded the pure compound $7(6.31 \mathrm{~g}, 87 \%)$ as a colorless oil; $R_{f}=0.4$ $\left(\mathrm{SiO}_{2}, 30 \%\right.$ EtOAc in PE); $[\alpha]_{\mathrm{D}}{ }^{26}-15.1\left(\right.$ c $\left.1.8, \mathrm{CHCl}_{3}\right)$.

IR (neat): $v_{\max }=2933,2378,2115,1513,1463,1248,1037,832,678$ $\mathrm{cm}^{-1}$.

${ }^{1} \mathrm{H}$ NMR $\left(300 \mathrm{MHz}, \mathrm{CDCl}_{3}\right): \delta=7.25(\mathrm{~d}, J=8.6 \mathrm{~Hz}, 2 \mathrm{H}), 6.87(\mathrm{~d}, J=8.6$ $\mathrm{Hz}, 2 \mathrm{H}), 4.41(\mathrm{q}, J=8.4 \mathrm{~Hz}, 2 \mathrm{H}), 4.06(\mathrm{p}, J=6.0 \mathrm{~Hz}, 1 \mathrm{H}), 3.81(\mathrm{~s}, 3 \mathrm{H})$, $3.52(\mathrm{t}, J=6.5 \mathrm{~Hz}, 2 \mathrm{H}), 3.04(\mathrm{~m}, 1 \mathrm{H}), 2.75(\mathrm{t}, J=4.5 \mathrm{~Hz}, 1 \mathrm{H}), 2.44(\mathrm{dd}$, $J=5.0,2.7 \mathrm{~Hz}, 1 \mathrm{H}), 1.85(\mathrm{q}, J=6.4 \mathrm{~Hz}, 2 \mathrm{H}), 1.68(\mathrm{t}, J=5.6 \mathrm{~Hz}, 2 \mathrm{H})$, $0.88(\mathrm{~s}, 9 \mathrm{H}), 0.06(\mathrm{~s}, 6 \mathrm{H})$.

${ }^{13} \mathrm{C}$ NMR $\left(125 \mathrm{MHz}, \mathrm{CDCl}_{3}\right): \delta=159.3,130.8,129.5,114.0,72.8,67.6$, $66.7,55.5,49.4,47.0,40.6,37.3,26.0,18.2,-4.3,-4.5$.

MS (ESI): $m / z=389[\mathrm{M}+\mathrm{Na}]^{+}$.

HRMS: $m / z$ calcd for $\mathrm{C}_{20} \mathrm{H}_{34} \mathrm{O}_{4} \mathrm{SiNa}[\mathrm{M}+\mathrm{Na}]^{+}$: 389.2119; found: 389.2124 .

\section{(4R,6S)-6-[(tert-Butyldimethylsilyl)oxy]-8-[(4-methoxybenzyl)- oxy]-1-(trimethylsilyl)oct-1-yn-4-ol (8)}

To a stirred solution of TMS-acetylene ( $3 \mathrm{~mL}, 43.5 \mathrm{mmol}$ ) in anhydrous THF $(40 \mathrm{~mL})$ was added $n$-BuLi $(23.84 \mathrm{~mL}, 1.6 \mathrm{M}$ in hexane $38.14 \mathrm{mmol})$ at $-78{ }^{\circ} \mathrm{C}$ under argon, and the mixture was stirred for $30 \mathrm{~min} . \mathrm{BF}_{3} \cdot \mathrm{OEt}_{2}(0.7 \mathrm{~mL}, 50 \mathrm{mmol})$ was added to the reaction mixture, and it was stirred for $15 \mathrm{~min}$. Compound 7 (dissolved in THF $2 \times$ $10 \mathrm{~mL} ; 4 \mathrm{~g}, 10.9 \mathrm{mmol}$ ) was added via cannula to the above reaction mixture, and the resultant mixture was stirred at $-78^{\circ} \mathrm{C}$. After completion of reaction (TLC), aqueous $\mathrm{NH}_{4} \mathrm{Cl}(40 \mathrm{~mL})$ was added, and the reaction mixture allowed to reach $\mathrm{rt}$. The mixture was extracted with EtOAc $(2 \times 150 \mathrm{~mL})$, the combined organic layers were washed with $\mathrm{H}_{2} \mathrm{O}(100 \mathrm{~mL})$ and brine $(50 \mathrm{~mL})$, dried over $\mathrm{Na}_{2} \mathrm{SO}_{4}$, filtered, and concentrated under vacuum. Purification of the crude product by column chromatography $\left(\mathrm{SiO}_{2}, 20 \%\right.$ EtOAc in hexane) afforded pure compound $8(4.30 \mathrm{~g}, 85 \%)$ as a colorless oil; $R_{f}=0.6\left(\mathrm{SiO}_{2}, 30 \%\right.$ EtOAc in $\mathrm{PE}) ;[\alpha]_{\mathrm{D}}^{23}+1.2\left(\mathrm{c} 1.4, \mathrm{CHCl}_{3}\right)$.

IR (neat): $v_{\max }=3462,2928,2174,1612,1512,1249,1090,840,651$ $\mathrm{cm}^{-1}$.

${ }^{1} \mathrm{H}$ NMR $\left(400 \mathrm{MHz}, \mathrm{CDCl}_{3}\right): \delta=7.24(\mathrm{~d}, J=8.4 \mathrm{~Hz}, 2 \mathrm{H}), 6.88(\mathrm{~d}, J=8.7$ $\mathrm{Hz}, 2 \mathrm{H}), 4.42$ (d, J = 1.5 Hz, $2 \mathrm{H}), 4.08(\mathrm{~m} 1 \mathrm{H}), 3.87(\mathrm{~m}, 1 \mathrm{H}), 3.80(\mathrm{~s}, 3$ H), $3.50(\mathrm{t}, J=5 \mathrm{~Hz}, 2 \mathrm{H}), 3.00(\mathrm{br} \mathrm{s}, 1 \mathrm{H}), 2.40(\mathrm{dd}, J=6.1,3.3 \mathrm{~Hz}, 2 \mathrm{H})$, 1.87-1.78 (m, $3 \mathrm{H}), 1.64(\mathrm{~m}, 2 \mathrm{H}), 0.88(\mathrm{~s}, 9 \mathrm{H}), 0.15$ (s, $9 \mathrm{H}), 0.09$ (s, 3 $\mathrm{H}), 0.08$ (s, $3 \mathrm{H})$.

${ }^{13} \mathrm{C}$ NMR $\left(125 \mathrm{MHz}, \mathrm{CDCl}_{3}\right): \delta=159.4,130.6,129.5,114.0,103.6,87.4$, 72.9, 69.7, 68.7, 66.6, 55.46, 42.7, 37.6, 29.2, 26.0, 18.1, 0.3, -4.1, -4.4. MS (ESI): $m / z=465[\mathrm{M}+\mathrm{H}]^{+}$.

HRMS: $m / z$ calcd for $\mathrm{C}_{25} \mathrm{H}_{45} \mathrm{O}_{4} \mathrm{Si}_{2}[\mathrm{M}+\mathrm{H}]^{+}: 465.2851$; found: 465.2856 .

\section{(3S,5R)-1-[(4-Methoxybenzyl)oxy]oct-7-yne-3,5-diol (9)}

To a solution of compound 8 ( $3.0 \mathrm{~g}, 6.4 \mathrm{mmol})$ in anhydrous THF (30 $\mathrm{mL}$ ) at $0{ }^{\circ} \mathrm{C}$, was added $\operatorname{TBAF}(32 \mathrm{~mL}, 32 \mathrm{mmol}, 1 \mathrm{M})$ and the mixture was stirred for $30 \mathrm{~min}$ at $0{ }^{\circ} \mathrm{C}$ then $30 \mathrm{~min}$ at rt. After completion of reaction (TLC), aqueous $\mathrm{NH}_{4} \mathrm{Cl}(20 \mathrm{~mL})$ was added, and the mixture extracted with EtOAc $(2 \times 100 \mathrm{~mL})$. The combined organic extracts were washed with $\mathrm{H}_{2} \mathrm{O}(20 \mathrm{~mL})$, brine $(20 \mathrm{~mL})$, and dried over $\mathrm{Na}_{2} \mathrm{SO}_{4}$. After filtration, the solvent was removed under vacuum, and the crude product was purified by column chromatography $\left(\mathrm{SiO}_{2}, 25 \%\right.$ EtOAc in hexane) to afford pure compound $\mathbf{9}(1.59 \mathrm{~g}, 89 \%)$ as a colorless oil; $R_{f}=0.6\left(\mathrm{SiO}_{2}, 40 \%\right.$ EtOAc in PE); $[\alpha]_{\mathrm{D}}^{23}+6.5\left(c 1.8, \mathrm{CHCl}_{3}\right)$.

IR (neat): $v_{\max }=3396$ (br), 3291 (br), 2922, 2312, 1611, 1512, 1245, $1086,1032,821,644 \mathrm{~cm}^{-1}$.

${ }^{1} \mathrm{H}$ NMR $\left(400 \mathrm{MHz}, \mathrm{CDCl}_{3}\right): \delta=7.24(\mathrm{~d}, J=8.5 \mathrm{~Hz}, 2 \mathrm{H}), 6.88(\mathrm{~d}, J=8.5$ $\mathrm{Hz}, 2 \mathrm{H}), 4.44$ (s, $2 \mathrm{H}), 4.09-3.97(\mathrm{~m}, 3 \mathrm{H}), 3.88(\mathrm{~m}, 1 \mathrm{H}), 3.79(\mathrm{~s}, 3 \mathrm{H})$, 3.72-3.60 (m, $2 \mathrm{H}), 2.44-2.29(\mathrm{~m}, 2 \mathrm{H}), 2.03(\mathrm{t}, J=2.7 \mathrm{~Hz}, 1 \mathrm{H}), 1.72$ $(\mathrm{m}, 4 \mathrm{H})$.

${ }^{13} \mathrm{C}$ NMR $\left(125 \mathrm{MHz}, \mathrm{CDCl}_{3}\right): \delta=159.4,129.9,129.5,114.0,81.1,73.1$, 72.3, 70.7, 70.6, 68.6, 55.4, 42.0, 36.9, 27.5 .

MS (ESI): $m / z=301[\mathrm{M}+\mathrm{Na}]^{+}$.

HRMS: $m / z$ calcd for $\mathrm{C}_{16} \mathrm{H}_{22} \mathrm{O}_{4} \mathrm{Na}[\mathrm{M}+\mathrm{Na}]^{+}$: 301.1410; found: 301.1419 .

(4S,6R)-4-\{2-[(4-Methoxybenzyl)oxy]ethyl\}-2,2-dimethyl-6-(prop2-yn-1-yl)-1,3-dioxane (5)

To a solution of diol $\mathbf{9}(1.0 \mathrm{~g}, 3.6 \mathrm{mmol})$ in anhydrous $\mathrm{CH}_{2} \mathrm{Cl}_{2}(10 \mathrm{~mL})$ at $0{ }^{\circ} \mathrm{C}$ was added 2,2-dimethoxypropane $(0.65 \mathrm{~mL}, 5.38 \mathrm{mmol})$ and CSA (417 mg, $1.79 \mathrm{mmol}$ ), and the mixture was stirred for $2 \mathrm{~h}$. After completion of reaction (TLC), aqueous $\mathrm{NaHCO}_{3}(5 \mathrm{~mL})$ was added, and the mixture was extracted with EtOAc $(3 \times 20 \mathrm{~mL})$. The combined organic layers were washed with $\mathrm{H}_{2} \mathrm{O}(10 \mathrm{~mL})$, brine $(10 \mathrm{~mL})$, and dried over $\mathrm{Na}_{2} \mathrm{SO}_{4}$. After filtration, evaporation of the organic extract under vacuum gave crude product that, on purification by column chromatography $\left(\mathrm{SiO}_{2}, 12 \%\right.$ EtOAc in hexane), afforded the pure 5 (919 mg, $92 \%)$ as a colorless oil; $R_{f}=0.3\left(\mathrm{SiO}_{2}, 30 \%\right.$ EtOAc in PE); $[\alpha]_{\mathrm{D}}{ }^{26}-26(c$ 2.2, $\mathrm{CHCl}_{3}$ ).

IR (neat): $v_{\max }=3394,2994,2377,2173,1512,1245,1093,816,648$ $\mathrm{cm}^{-1}$.

${ }^{1} \mathrm{H}$ NMR $\left(400 \mathrm{MHz}, \mathrm{CDCl}_{3}\right): \delta=7.25(\mathrm{~d}, J=8.0 \mathrm{~Hz}, 2 \mathrm{H}), 6.88(\mathrm{~d}, J=8.0$ $\mathrm{Hz}, 2 \mathrm{H}), 4.45(\mathrm{~d}, J=11.6 \mathrm{~Hz}, 1 \mathrm{H}), 4.42(\mathrm{~d}, J=11.6 \mathrm{~Hz}, 1 \mathrm{H}), 4.07-3.93$ (m, $2 \mathrm{H}), 3.81$ (s, $3 \mathrm{H}), 3.59-3.48(\mathrm{~m}, 2 \mathrm{H}), 2.47(\mathrm{dd}, J=2.8,5.2 \mathrm{~Hz}, 1 \mathrm{H})$, $2.42(\mathrm{dd}, J=2.8,5.2 \mathrm{~Hz}, 1 \mathrm{H}), 2.00(\mathrm{t}, J=2.7 \mathrm{~Hz}, 1 \mathrm{H}), 1.79-1.71(\mathrm{~m}, 2$ $\mathrm{H}), 1.43(\mathrm{~s}, 3 \mathrm{H}), 1.38$ (s, $3 \mathrm{H}), 1.28-1.13(\mathrm{~m}, 2 \mathrm{H})$. 
${ }^{13} \mathrm{C}$ NMR $\left(100 \mathrm{MHz}, \mathrm{CDCl}_{3}\right): \delta=159.3,130.8,129.4,114.0,99.0,80.5$, $72.8,70.5,68.0,66.2,66.0,55.4,36.7,36.3,30.3,26.4,20.0$.

MS (ESI): $m / z=341[\mathrm{M}+\mathrm{Na}]^{+}$.

HRMS: $m / z$ calcd for $\mathrm{C}_{19} \mathrm{H}_{26} \mathrm{O}_{4} \mathrm{Na}[\mathrm{M}+\mathrm{Na}]^{+}$: 341.1723; found: 341.1719 .

\section{(R)-8-(Benzyloxy)-1-(trimethylsilyl)oct-1-yn-4-ol (11)}

A stirred solution of TMS-acetylene $(8.5 \mathrm{~mL}, 60.6 \mathrm{mmol})$ in anhydrous THF $(60 \mathrm{~mL})$ was treated with $n$-BuLi $(22.75 \mathrm{~mL}, 1.6 \mathrm{M}$ in hexane, $36.44 \mathrm{mmol}$ ) at $-78{ }^{\circ} \mathrm{C}$ under nitrogen, and the mixture was stirred for $30 \mathrm{~min}$. $\mathrm{BF}_{3} \cdot \mathrm{OEt}_{2}(1.56 \mathrm{~mL}, 12.1 \mathrm{mmol})$ was then added and the mixture was stirred for $30 \mathrm{~min}$. Epoxide $10(5.0 \mathrm{~g}, 24.2 \mathrm{mmol})$ dissolved in anhydrous THF $(2 \times 10 \mathrm{~mL})$ was added via cannula, and the mixture was stirred for $2 \mathrm{~h}$ at $-78^{\circ} \mathrm{C}$. A saturated solution of aqueous $\mathrm{NH}_{4} \mathrm{Cl}(80 \mathrm{~mL})$ was added, and the mixture was allowed to reach rt. The reaction mixture was extracted with EtOAc $(2 \times 250 \mathrm{~mL})$, and the combined organic extracts were washed with brine $(50 \mathrm{~mL})$ and dried over anhydrous $\mathrm{Na}_{2} \mathrm{SO}_{4}$. Filtration and evaporation of the organic solvents under reduced pressure provided a crude resisdue that, on purification by column chromatography $\left(\mathrm{SiO}_{2}, 8 \% \mathrm{EtOAc} /\right.$ hexane), furnished compound 11 as a colorless oil $(6.26 \mathrm{~g}, 85 \%) \cdot R_{f}=0.3\left(\mathrm{SiO}_{2}, 10 \%\right.$ EtOAc/hexane); $[\alpha]_{D}{ }^{27}-12.1$ (c 1.9, $\mathrm{CHCl}_{3}$ ).

IR (neat): $v_{\max }=3423$ (br), 2937,2861, 1495, 1432, 1094, 1027, 843, $747,697,632 \mathrm{~cm}^{-1}$.

${ }^{1} \mathrm{H}$ NMR $\left(500 \mathrm{MHz}, \mathrm{CDCl}_{3}\right): \delta=7.29-7.25(\mathrm{~m}, 4 \mathrm{H}), 7.23-7.18(\mathrm{~m}, 1 \mathrm{H})$, $4.43(\mathrm{~s}, 2 \mathrm{H}), 3.66(\mathrm{~m}, 1 \mathrm{H}), 3.41(\mathrm{t}, J=6.5 \mathrm{~Hz}, 2 \mathrm{H}), 2.37$ (dd, $J=17,5.0$, $\mathrm{Hz}, 1 \mathrm{H}), 2.27$ (dd, J = 17, 2.0, Hz, $1 \mathrm{H}$ ), 1.99 (br s, $1 \mathrm{H}), 1.63-1.53$ (m, 2 $\mathrm{H}), 1.51-1.42(\mathrm{~m}, 3 \mathrm{H}), 1.41-1.32(\mathrm{~m}, 1 \mathrm{H}), 0.09(\mathrm{~s}, 9 \mathrm{H})$.

${ }^{13} \mathrm{C}$ NMR $\left(100 \mathrm{MHz}, \mathrm{CDCl}_{3}\right): \delta=138.7,128.6,127.8,127.7,103.5,87.8$, 73.1, 70.4, 70.0, 36.1, 29.8, 29.0, 22.5, 0.3.

MS (ESI): $m / z=305[\mathrm{M}+\mathrm{H}]^{+}$.

HRMS: $m / z$ calcd for $\mathrm{C}_{18} \mathrm{H}_{29} \mathrm{O}_{2} \mathrm{Si}[\mathrm{M}+\mathrm{H}]^{+}$: 305.1931; found: 305.1931 .

\section{(R)-8-(Benzyloxy)oct-1-yn-4-ol (12)}

Compound 11 (5.0 g, $16.4 \mathrm{mmol}$ ) was dissolved in anhydrous $\mathrm{MeOH}$ $(150 \mathrm{~mL})$ and treated with anhydrous $\mathrm{K}_{2} \mathrm{CO}_{3}(4.53 \mathrm{~g}, 32.8 \mathrm{mmol})$ at $0{ }^{\circ} \mathrm{C}$ under nitrogen. The reaction mixture was stirred at $\mathrm{rt}$ for $2 \mathrm{~h}$ and quenched with $\mathrm{H}_{2} \mathrm{O}(50 \mathrm{~mL})$. $\mathrm{MeOH}$ was removed under reduced pressure, and the aqueous layer was extracted with EtOAc $(2 \times 80 \mathrm{~mL})$. The combined organic extracts were washed with water $(2 \times 40 \mathrm{~mL})$ and brine $(20 \mathrm{~mL})$. After drying over anhydrous $\mathrm{Na}_{2} \mathrm{SO}_{4}$ and filtering, the solvent was evaporated under reduced pressure to give a residue that, on purification by flash chromatography $\left(\mathrm{SiO}_{2}, 20 \% \mathrm{EtOAc} /\right.$ hexane), afforded 12 as a colorless oil $(3.20 \mathrm{~g}, 84 \%) . R_{f}=0.6\left(\mathrm{SiO}_{2}, 20 \% \mathrm{EtOAC} /\right.$ hexane); $[\alpha]_{D}^{30}-5.4\left(c 2.6, \mathrm{CHCl}_{3}\right)$.

IR (neat): $v_{\max }=3418$ (br) 3305, 2937, 2862, 2117, 1495, 1432, 1248, $1094,748,665,632 \mathrm{~cm}^{-1}$.

${ }^{1} \mathrm{H}$ NMR (400 MHz, $\mathrm{CDCl}_{3}$ ): $\delta=7.28-7.18(\mathrm{~m}, 5 \mathrm{H}), 4.43(\mathrm{~s}, 2 \mathrm{H}), 3.68$ $(\mathrm{m}, 1 \mathrm{H}), 3.41(\mathrm{t}, J=6.4 \mathrm{~Hz}, 2 \mathrm{H}), 2.30$ (ddd, $J=16.5,4.8,2.4 \mathrm{~Hz}, 1 \mathrm{H})$, 2.30 (ddd, $J=16.5,6.8,2.8 \mathrm{~Hz}, 1 \mathrm{H}$ ), 1.97 (t, J=2.8 Hz, $1 \mathrm{H}$ ), 1.92 (br s, $1 \mathrm{H}), 1.62-1.34(\mathrm{~m}, 6 \mathrm{H})$.

${ }^{13} \mathrm{C}$ NMR $\left(125 \mathrm{MHz}, \mathrm{CDCl}_{3}\right): \delta=138.59,128.45,127.75,127.63,81.10$, 72.99, 70.85, 70.27, 69.79, 35.99, 29.63, 27.43, 22.41 .

MS (ESI): $m / z=233[\mathrm{M}+\mathrm{H}]^{+}$.

HRMS: $m / z$ calcd for $\mathrm{C}_{15} \mathrm{H}_{21} \mathrm{O}_{2}[\mathrm{M}+\mathrm{H}]^{+}$: 233.1541; found: 233.1536 .

\section{(R)-\{[8-(Benzyloxy)oct-1-yn-4-yl]oxy\}(tert-butyl)dimethylsilane} (13)

Compound 12 (3.2.0 g, $13.7 \mathrm{mmol}$ ) was dissolved in anhydrous $\mathrm{CH}_{2} \mathrm{Cl}_{2}$ $(60 \mathrm{~mL})$ and treated with 2,6-lutidine $(3.68 \mathrm{~mL}, 34.4 \mathrm{mmol})$ and TBSOTf ( $4.96 \mathrm{~mL}, 20 \mathrm{mmol})$ at $0{ }^{\circ} \mathrm{C}$. The reaction mixture was stirred for $2 \mathrm{~h}$, quenched with saturated aqueous $\mathrm{NaHCO}_{3}(20 \mathrm{~mL})$ and extracted with EtOAc $(2 \times 120 \mathrm{~mL})$. The combined organic extracts were washed with saturated aqueous $\mathrm{CuSO}_{4}(2 \times 40 \mathrm{~mL})$, water $(2 \times 40 \mathrm{~mL})$, and brine $(50 \mathrm{~mL})$. After drying the organic extract over anhydrous $\mathrm{Na}_{2} \mathrm{SO}_{4}$ and filtering, the solvent was evaporated under reduced pressure, and the crude product was purified by column chromatography $\left(\mathrm{SiO}_{2}, 20 \% \mathrm{EtOAc} /\right.$ hexane), which afforded the title compound $\mathbf{1 3}$ as a colorless oil (4.32 g, 91\%). $R_{f}=0.3\left(\mathrm{SiO}_{2}, 20 \%\right.$ EtOAc/hexane); $[\alpha]_{\mathrm{D}}{ }^{30}$ $+12.5\left(\right.$ c $\left.1.8, \mathrm{CHCl}_{3}\right)$.

IR (neat): $v_{\max }=3311,2930,2856,2349,1389,1253,1102,834,774$, $698 \mathrm{~cm}^{-1}$.

${ }^{1} \mathrm{H} \mathrm{NMR}\left(400 \mathrm{MHz}, \mathrm{CDCl}_{3}\right): \delta=7.28-7.18(\mathrm{~m}, 5 \mathrm{H}), 4.43(\mathrm{~s}, 2 \mathrm{H}), 3.72$ $(\mathrm{m}, 1 \mathrm{H}), 3.41(\mathrm{t}, J=6.6 \mathrm{~Hz}, 2 \mathrm{H}), 2.26-2.21(\mathrm{~m}, 2 \mathrm{H}), 1.89(\mathrm{t}, J=2.7 \mathrm{~Hz}$, $1 \mathrm{H}), 1.61-1.52(\mathrm{~m}, 3 \mathrm{H}), 1.51-1.41(\mathrm{~m}, 2 \mathrm{H}), 1.29(\mathrm{~m}, 1 \mathrm{H}), 0.81(\mathrm{~s}, 9$ $\mathrm{H}), 0.00(\mathrm{~s}, 3 \mathrm{H}),-0.01(\mathrm{~s}, 3 \mathrm{H})$.

${ }^{13} \mathrm{C}$ NMR $\left(100 \mathrm{MHz}, \mathrm{CDCl}_{3}\right): \delta=138.9,128.5,127.8,127.7,81.9,73.0$, 71.5, 70.5, 70.0, 36.6, 30.0, 27.6, 26.1, 22.1, 18.3, -4.3, -4.4.

MS (ESI): $m / z=347[\mathrm{M}+\mathrm{H}]^{+}$.

HRMS: $m / z$ calcd for $\mathrm{C}_{19} \mathrm{H}_{26} \mathrm{O}_{4}[\mathrm{M}+\mathrm{H}]^{+}$: 347.2401; found: 347.2401.

(R)-5-[4-(Benzyloxy)butyl]-2,2,3,3,16,16-hexamethyl-15,15-diphenyl-4,14-dioxa-3,15-disilaheptadec-7-yn-9-one (15)

To a solution of $\mathbf{1 3}$ (4.5 g, $13 \mathrm{mmol})$ in dry THF (70 mL) under argon, $n$-BuLi (1.6 M in hexane, $9.75 \mathrm{~mL}, 15.6 \mathrm{mmol}$ ) was added at $-78{ }^{\circ} \mathrm{C}$. After stirring for $30 \mathrm{~min}$ at $-78{ }^{\circ} \mathrm{C}$, aldehyde 20 ( $\left.7.48 \mathrm{~g}, 16.9 \mathrm{mmol}\right)$ dissolved in anyhydrous THF $(20 \mathrm{~mL})$ was added dropwise via cannula. After stirring for $2.5 \mathrm{~h}$ at $-78{ }^{\circ} \mathrm{C}$, saturated aqueous $\mathrm{NH}_{4} \mathrm{Cl}(50 \mathrm{~mL})$ was added to the reaction mixture, and the temperature was allowed to rise to rt slowly. The reaction mixture was extracted with EtOAc (2 $\times 150 \mathrm{~mL}$ ), and the combined organic extracts were washed with water $(2 \times 40 \mathrm{~mL})$ and brine $(30 \mathrm{~mL})$. After drying the organic extracts with anhydrous $\mathrm{Na}_{2} \mathrm{SO}_{4}$ and filtering, the solvent was evaporated under reduced pressure to give a residue that was subjected to flash chromatography $\left(\mathrm{SiO}_{2}, 12 \% \mathrm{EtOAc} /\right.$ hexane) to afford compound $\mathbf{1 4}$ as a colorless oil $(7.14 \mathrm{~g}, 80 \%)$.

To a solution of compound $\mathbf{1 4}$ (7.14 g, $10.3 \mathrm{mmol})$ in $\mathrm{CH}_{2} \mathrm{Cl}_{2}(100 \mathrm{~mL})$ at $0{ }^{\circ} \mathrm{C}$ was added $\mathrm{NaHCO}_{3}(2.2 \mathrm{~g}, 25.7 \mathrm{mmol})$ under nitrogen. Then $\operatorname{DMP}(8.8 \mathrm{~g}, 20 \mathrm{mmol}$ ) was added portionwise at the same temperature. The reaction mixture was allowed to warm to rt and stirred for 4 h. Saturated aqueous $\mathrm{Na}_{2} \mathrm{~S}_{2} \mathrm{O}_{3}(50 \mathrm{~mL})$ and saturated aqueous $\mathrm{NaHCO}_{3}$ $(30 \mathrm{~mL})$ were added, and the resultant biphasic mixture was stirred for $50 \mathrm{~min}$ and then extracted with EtOAc $(2 \times 150 \mathrm{~mL})$. The combined organic extracts were washed with water $(50 \mathrm{~mL})$ and brine $(40 \mathrm{~mL})$. The organic extracts wer dried over anhydrous $\mathrm{Na}_{2} \mathrm{SO}_{4}$, filtered, and concentrated under reduced pressure. Purification of the residue by column chromatography $\left(\mathrm{SiO}_{2}, 10 \% \mathrm{EtOAc} /\right.$ hexane) afforded compound 15 as a colorless oil $(6.76 \mathrm{~g}, 95 \%) . R_{f}=0.5\left(\mathrm{SiO}_{2}, 20 \% \mathrm{EtOAc} /\right.$ hexane); $[\alpha]_{D}^{30}+3.2\left(c 1.5, \mathrm{CHCl}_{3}\right)$.

IR (neat): $v_{\max }=2931,2856,2214,1716,1427,1247,1110,849,701$, $606 \mathrm{~cm}^{-1}$.

${ }^{1} \mathrm{H}$ NMR $\left(400 \mathrm{MHz}, \mathrm{CDCl}_{3}\right): \delta=7.63-7.57(\mathrm{~m}, 4 \mathrm{H}), 7.38-7.26(\mathrm{~m}, 8 \mathrm{H})$, 7.25-7.16 (m, $2 \mathrm{H}), 4.43(\mathrm{~s}, 2 \mathrm{H}), 3.78$ (pent, $J=6 \mathrm{~Hz}, 1 \mathrm{H}), 3.59(\mathrm{t}, J=$ $6.2 \mathrm{~Hz}, 2 \mathrm{H}), 3.40(\mathrm{t}, J=6.4 \mathrm{~Hz}, 2 \mathrm{H}), 2.45(\mathrm{t}, J=7.2 \mathrm{~Hz}, 2 \mathrm{H}), 2.41$ (d, $J=$ $6 \mathrm{~Hz}, 2 \mathrm{H}$ ), 1.69 (pent, $J=7.2 \mathrm{~Hz}, 2 \mathrm{H}), 1.58-1.47$ (m, $6 \mathrm{H}), 1.44-1.26$ (m, $2 \mathrm{H}), 0.98$ (s, $9 \mathrm{H}), 0.82(\mathrm{~s}, 9 \mathrm{H}), 0.01(\mathrm{~s}, 3 \mathrm{H}), 0.00$ (s, $3 \mathrm{H})$. 
${ }^{13} \mathrm{C}$ NMR $\left(100 \mathrm{MHz}, \mathrm{CDCl}_{3}\right): \delta=188.1,138.8,135.7,134.1,129.8$, $128.5,127.8,127.8,127.7,91.4,82.4,73.1,70.5,70.4,63.6,45.4,37.0$, $31.9,29.9,28.0,27.0,26.0,22.0,20.7,19.4,18.21,-4.3,-4.4$.

MS (ESI): $m / z=685[\mathrm{M}+\mathrm{H}]^{+}$.

HRMS: $m / z$ calcd for $\mathrm{C}_{42} \mathrm{H}_{61} \mathrm{O}_{4} \mathrm{Si}_{2}[\mathrm{M}+\mathrm{H}]^{+}$: 685.4103; found: 685.4107 .

\section{(5R,9S)-5-[4-(Benzyloxy)butyl]-2,2,3,3,16,16-hexamethyl-15,15- diphenyl-4,14-dioxa-3,15-disilaheptadec-7-yn-9-ol (16)}

Alkynone 15 (6.5 g, $9.48 \mathrm{mmol})$ was dissolved in $i-\mathrm{PrOH}(120 \mathrm{~mL})$ at $\mathrm{rt}$ and treated with $\mathrm{Ru}[(S, S)$-Tsdpen]( $p$-cymene) $(30 \mathrm{mg}, 948 \mu \mathrm{mol})$. After stirring for $36 \mathrm{~h}$ at $\mathrm{rt}$, the solvent was removed under reduced pressure to give the crude product, that on purification by column chromatography $\left(\mathrm{SiO}_{2}, 20 \%\right.$ EtOAc/hexane) afforded compound $\mathbf{1 6}$ as a colorless oil (5.4 g, 83\%). $R_{f}=0.3\left(\mathrm{SiO}_{2}, 20 \%\right.$ EtOAc/hexane); $[\alpha]_{\mathrm{D}}^{29}+5.09$ (c $16.5, \mathrm{CHCl}_{3}$ ).

IR (neat): $v_{\max }=3443$ (br), 2930, 2857, 2214, 1414, 1254, 1106, 824, $775,700,613 \mathrm{~cm}^{-1}$.

$\left.{ }^{1} \mathrm{H} \mathrm{NMR} \mathrm{(400} \mathrm{MHz,} \mathrm{CDCl}_{3}\right): \delta=7.63-7.57(\mathrm{~m}, 4 \mathrm{H}), 7.36-7.28(\mathrm{~m}, 6 \mathrm{H})$, $7.27(\mathrm{~m}, 3 \mathrm{H}), 7.23-7.17(\mathrm{~m}, 2 \mathrm{H}), 4.43(\mathrm{~s}, 2 \mathrm{H}), 4.24(\mathrm{~m}, 1 \mathrm{H}), 3.71$ (pent, 6.4 Hz), $3.60(\mathrm{t}, J=6.3 \mathrm{~Hz}, 2 \mathrm{H}), 3.40(\mathrm{t}, J=6.5 \mathrm{~Hz}, 2 \mathrm{H}), 2.27-$ $2.25(\mathrm{~m}, 2 \mathrm{H}), 1.72(\mathrm{~d}, J=4.8 \mathrm{~Hz}, 1 \mathrm{H}), 1.60-1.49(\mathrm{~m}, 7 \mathrm{H}), 1.45(\mathrm{~m}, 3$ H), 1.34-1.30 (m, $1 \mathrm{H}), 0.98(\mathrm{~s}, 9 \mathrm{H}), 0.81(\mathrm{~s}, 9 \mathrm{H}), 0.00$ (s, $3 \mathrm{H}),-0.01$ (s, $3 \mathrm{H})$.

${ }^{13} \mathrm{C}$ NMR $\left(100 \mathrm{MHz}, \mathrm{CDCl}_{3}\right): \delta=138.8,135.8,134.2,129.7,128.5$, 127.8, 127.7, 83.1, 82.7, 73.1, 71.1, 70.5, 63.9, 62.9, 3f8.0, 36.8, 32.4, $30.0,27.8,27.1,26.1,22.1,21.7,19.4,18.3,-4.2,-4.4$.

MS (ESI): $m / z=669\left[\mathrm{M}+\mathrm{NH}_{4}\right]^{+}$.

HRMS: $\mathrm{m} / z$ calcd for $\mathrm{C}_{42} \mathrm{H}_{66} \mathrm{O}_{4} \mathrm{Si}_{2} \mathrm{~N}\left[\mathrm{M}+\mathrm{NH}_{4}\right]^{+}:$704.4525; found: 704.4524 .

\section{(5R,9S)-5-[4-(Benzyloxy)butyl]-2,2,3,3,16,16-hexamethyl-15,15- diphenyl-4,14-dioxa-3,15-disilaheptadec-7-yn-9-ol (17)}

Compound 16 (6.0 g, $8.7 \mathrm{mmol}$ ) was dissolved in anhydrous $\mathrm{CH}_{2} \mathrm{Cl}_{2}$ $(100 \mathrm{~mL})$ and treated with 2,6-lutidine $(2 \mathrm{~mL}, 17.4 \mathrm{mmol})$ and TBSOTf (3.12 mL, $13.1 \mathrm{mmol}$ ) at $0{ }^{\circ} \mathrm{C}$. The reaction mixture was stirred for $1 \mathrm{~h}$, quenched with saturated aqueous $\mathrm{NaHCO}_{3}(20 \mathrm{~mL})$, and extracted with EtOAc $(2 \times 150 \mathrm{~mL})$. The combined organic extracts were washed with saturated aqueous $\mathrm{CuSO}_{4}(2 \times 50 \mathrm{~mL})$, water $(50 \mathrm{~mL})$, and brine $(50 \mathrm{~mL})$. After drying the organic extract over anhydrous $\mathrm{Na}_{2} \mathrm{SO}_{4}$ and filtering, the solvent was evaporated under reduced pressure, and the residue was purified by column chromatography $\left(\mathrm{SiO}_{2}, 20 \% \mathrm{EtOAC} /\right.$ hexane) to afford 17 as a colorless oil $(6.69 \mathrm{~g}, 96 \%) . R_{f}=0.3\left(\mathrm{SiO}_{2}, 20 \%\right.$ EtOAc/hexane $) ;[\alpha]_{\mathrm{D}}{ }^{30}+5.2\left(\right.$ c $\left.3.3, \mathrm{CHCl}_{3}\right)$.

IR (neat): $v_{\max }=2937,2858,2349,1463,1370,1254,1102,833,772$, $701,613 \mathrm{~cm}^{-1}$

$\left.{ }^{1} \mathrm{H} \mathrm{NMR} \mathrm{(500} \mathrm{MHz,} \mathrm{CDCl}_{3}\right): \delta=7.67(\mathrm{~m}, 4 \mathrm{H}), 7.42-7.33(\mathrm{~m}, 8 \mathrm{H}), 7.29-$ $7.24(\mathrm{~m}, 2 \mathrm{H}), 4.50(\mathrm{~s}, 2 \mathrm{H}), 4.30(\mathrm{~m}, 1 \mathrm{H}), 3.74(\mathrm{~m}, 1 \mathrm{H}), 3.65(\mathrm{t}, J=6.2$ $\mathrm{Hz}, 2 \mathrm{H}$ ), 3.46 (t, $J=6.6 \mathrm{~Hz}, 2 \mathrm{H}), 2.34$ (ddd, $J=16.5,5.0,2.0 \mathrm{~Hz} 1 \mathrm{H}$ ), 2.28 (ddd, $J=16.5,7.0,2.0 \mathrm{~Hz} 1 \mathrm{H}) 1.67-1.57(\mathrm{~m}, 7 \mathrm{H}), 1.53-1.45(\mathrm{~m}, 4$ H), 1.38-1.33 (m, $1 \mathrm{H}), 1.04(\mathrm{~s}, 9 \mathrm{H}), 0.90$ (s, $9 \mathrm{H}), 0.88$ (s, $9 \mathrm{H}), 0.11$ (s, $3 \mathrm{H}), 0.09(\mathrm{~s}, 3 \mathrm{H}), 0.06(\mathrm{~s}, 3 \mathrm{H}), 0.04(\mathrm{~s}, 3 \mathrm{H})$.

${ }^{13} \mathrm{C}$ NMR $\left(125 \mathrm{MHz}, \mathrm{CDCl}_{3}\right): \delta=138.9,135.8,134.3,129.7,128.5$, 127.8, 127.7, 83.8, 81.5, 73.1, 71.3, 70.6, 64.1, 63.4, 39.0, 36.6, 32.5, $30.1,28.0,27.1,26.1,26.1,22.1,22.0,19.4,18.5,18.3,-4.2,-4.5,-4.7$. MS (ESI): $m / z=818\left[\mathrm{M}+\mathrm{NH}_{4}\right]^{+}$.

HRMS: $\mathrm{m} / z$ calcd for $\mathrm{C}_{48} \mathrm{H}_{80} \mathrm{O}_{4} \mathrm{Si}_{3} \mathrm{~N}\left[\mathrm{M}+\mathrm{NH}_{4}\right]^{+}$: 818.5390; found: 818.5403 .
(5R,9S)-5-[(tert-Butyldimethylsilyl)oxy]-13-[(tert-butyldiphenylsilyl)oxy]tridecane-1,9-diol (18)

A stirred solution of compound $\mathbf{1 7}(6.5 \mathrm{~g}, 8.1 \mathrm{mmol})$ in anhydrous EtOAc (100 mL) was treated with $10 \% \mathrm{Pd} / \mathrm{C}(86 \mathrm{mg}, 20 \% \mathrm{w} / \mathrm{w})$ and hydrogenated using a hydrogen-filled balloon at $\mathrm{rt}$ for $5 \mathrm{~h}$. The reaction mixture was filtered through a Celite plug, and the plug was washed with EtOAc $(2 \times 100 \mathrm{~mL})$. The combined filtrate and washings were concentrated under reduced pressure to give the crude product, which was purified by silica gel column chromatography $\left(\mathrm{SiO}_{2}, 100\right.$ 200 mesh, 10\% EtOAc/hexane) to afford compound $\mathbf{1 8}(4.92 \mathrm{~g} \mathrm{85 \% )}$ as a colorless oil. $R_{f}=0.5\left(\mathrm{SiO}_{2}, 20 \%\right.$ EtOAc/hexane $) ;[\alpha]_{\mathrm{D}}^{25}-2.3$ (c 1.8, $\mathrm{CHCl}_{3}$ ).

IR (neat): $v_{\max }=3071$ (br), 2929, 2856, 1413, 1390, 1253, 1108, 833, $772,700,613 \mathrm{~cm}^{-1}$.

${ }^{1} \mathrm{H}$ NMR $\left(500 \mathrm{MHz}, \mathrm{CDCl}_{3}\right): \delta=7.68-7.66(\mathrm{~m}, 4 \mathrm{H}), 7.44-7.34(\mathrm{~m}, 6 \mathrm{H})$, 3.66-3.60 (m, $6 \mathrm{H}), 1.59-1.53(\mathrm{~m}, 6 \mathrm{H}), 1.45-1.36(\mathrm{~m}, 12 \mathrm{H}), 1.04(\mathrm{~s}, 9$ $\mathrm{H}), 0.88(\mathrm{~s}, 9 \mathrm{H}), 0.87(\mathrm{~s}, 9 \mathrm{H}), 0.03(\mathrm{~s}, 6 \mathrm{H}), 0.03(\mathrm{~s}, 3 \mathrm{H}), 0.02(\mathrm{~s}, 3 \mathrm{H})$.

${ }^{13} \mathrm{C}$ NMR $\left(100 \mathrm{MHz}, \mathrm{CDCl}_{3}\right): \delta=135.8,134.3,129.7,127.8,72.5,72.4$, 64.2, 63.2, 37.6, 37.1, 36.9, 33.2, 33.1, 30.0, 29.8, 27.1, 26.2, 21.9, 21.6, $21.2,19.4,18.4,-4.2$

MS (ESI): $m / z=716[\mathrm{M}+\mathrm{H}]^{+}$.

HRMS: $m / z$ calcd for $\mathrm{C}_{41} \mathrm{H}_{78} \mathrm{O}_{4} \mathrm{Si}_{3} \mathrm{~N}\left[\mathrm{M}+\mathrm{NH}_{4}\right]^{+}$: 732.5232; found: 732.5233 .

(5S,9S,13S)-9,13-bis[(tert-Butyldimethylsilyl)oxy]-5-\{3-[(4R,6S)-6\{2-[(4-methoxybenzyl)oxy]ethyl\}-2,2-dimethyl-1,3-dioxan-4-yl]prop-1-yn-1-yl\}-2,2,3,3,20,20-hexamethyl-19,19-diphenyl-4,18dioxa-3,19-disilahenicosane (3)

Alcohol 18 (200 mg, $0.28 \mathrm{mmol}$ ) was dissolved in anhydrous $\mathrm{CH}_{2} \mathrm{Cl}_{2}$ $(10 \mathrm{~mL})$ and the solution cooled to $0{ }^{\circ} \mathrm{C} . \mathrm{NaHCO}_{3}(84 \mathrm{mg}, 0.97 \mathrm{mmol})$ and DMP (294 $\mathrm{mg}, 0.68 \mathrm{mmol}$ ) were added sequentially at the same temperature, and the resulting reaction mixture was stirred under nitrogen for $3 \mathrm{~h}$ at rt. Saturated aqueous $\mathrm{Na}_{2} \mathrm{~S}_{2} \mathrm{O}_{3}(10 \mathrm{~mL})$ and saturated aqueous $\mathrm{NaHCO}_{3}(10 \mathrm{~mL})$ were added to the reaction mixture, and the resultant biphasic mixture was stirred for $30 \mathrm{~min}$. The mixture was extracted with EtOAc $(2 \times 15 \mathrm{~mL})$, and the combined organic extracts were washed with water $(10 \mathrm{~mL})$ and brine $(10 \mathrm{~mL})$, dried over anhydrous $\mathrm{Na}_{2} \mathrm{SO}_{4}$, and filtered. The solvents were evaporated under reduced pressure to give aldehyde $\mathbf{4}\left(175 \mathrm{mg}, 88 \% ; R_{f}=0.4,10 \% \mathrm{EtOAc} /\right.$ hexane), which was used directly for the next reaction.

To a stirred solution of $\mathbf{5}$ (238 $\mathrm{mg}, 0.75 \mathrm{mmol})$ in dry THF ( $8 \mathrm{~mL})$ under nitrogen, $n$-BuLi (1.6 M in hexane, $0.4 \mathrm{~mL}, 0.625 \mathrm{mmol}$ ) was added at $-78{ }^{\circ} \mathrm{C}$. After stirring for $30 \mathrm{~min}$ at $-78^{\circ} \mathrm{C}$, crude aldehyde $4(175$ $\mathrm{mg}, 0.25 \mathrm{mmol})$ dissolved in THF $(2 \times 4 \mathrm{~mL})$ was added dropwise via cannula. After stirring for $45 \mathrm{~min}$ at $-78{ }^{\circ} \mathrm{C}$, saturated aqueous $\mathrm{NH}_{4} \mathrm{Cl}$ $(5 \mathrm{~mL}$ ) was added, and the temperature was allowed to raise to $\mathrm{rt}$ slowly. The reaction mixture was extracted with EtOAc $(2 \times 15 \mathrm{~mL})$ and the combined organic extracts washed with water $(10 \mathrm{~mL})$ and brine $(10 \mathrm{~mL})$. After drying the organic extracts over anhydrous $\mathrm{Na}_{2} \mathrm{SO}_{4}$ and filtering, the solvent was evaporated under reduced pressure to give a crude product that was subjected to flash chromatography $\left(\mathrm{SiO}_{2}, 15 \% \mathrm{EtOAc} /\right.$ hexane $)$ to afford 21 as a mixture of diastereomers as a colorless oil (218 $\mathrm{mg}, 85 \%)$.

To a solution of compound 21 (218 mg, $0.21 \mathrm{mmol})$ in $\mathrm{CH}_{2} \mathrm{Cl}_{2}(10 \mathrm{~mL})$ at $0{ }^{\circ} \mathrm{C}$ was added $\mathrm{NaHCO}_{3}(54 \mathrm{mg}, 0.63 \mathrm{mmol})$ under nitrogen, then DMP (178 $\mathrm{mg}, 0.42 \mathrm{mmol}$ ) was added portion-wise. The reaction mixture was warmed to rt and stirred for $4 \mathrm{~h}$. Saturated aqueous $\mathrm{Na}_{2} \mathrm{~S}_{2} \mathrm{O}_{3}(5 \mathrm{~mL})$ and saturated aqueous $\mathrm{NaHCO}_{3}(5 \mathrm{~mL})$ were added, and the resultant biphasic mixture was stirred for $10 \mathrm{~min}$ and then extracted with EtOAc $(2 \times 15 \mathrm{~mL})$. The combined organic extracts 
were washed with water $(10 \mathrm{~mL})$ and brine $(10 \mathrm{~mL})$, dried over anhydrous $\mathrm{Na}_{2} \mathrm{SO}_{4}$, filtered, and concentrated under reduced pressure. $\mathrm{Pu}-$ rification of the residue by column chromatography $\left(\mathrm{SiO}_{2}, 10 \% \mathrm{EtOAc} /\right.$ hexane) afforded the ketone as a colorless oil (164 mg, 76\%). $R_{f}=0.4$ $\left(\mathrm{SiO}_{2}, 20 \% \mathrm{EtOAc} / \mathrm{hexane}\right)$.

The purified alkynone ( $164 \mathrm{mg}, 0.16 \mathrm{mmol}$ ) was dissolved in $i$-PrOH $(4 \mathrm{~mL})$ at $\mathrm{rt}$ and treated with $\mathrm{Ru}[(S, S)$-Tsdpen](p-cymene) $(5 \mathrm{mg}, 8$ $\mu \mathrm{mol})$. After stirring for $48 \mathrm{~h}$ at $\mathrm{rt}$, the solvent was removed under reduced pressure to give that crude product that, on purification by flash chromatography $\left(\mathrm{SiO}_{2}, 15 \% \mathrm{EtOAc} /\right.$ hexane $)$, afforded the alcohol compound as a colorless oil ( $143 \mathrm{mg}, 87 \%) . R_{f}=0.6\left(\mathrm{SiO}_{2}, 20 \% \mathrm{EtOAc} /\right.$ hexane).

The purified alcohol ( $143 \mathrm{mg}, 0.14 \mathrm{mmol}$ ) was dissolved in anhydrous $\mathrm{CH}_{2} \mathrm{Cl}_{2}(5 \mathrm{~mL})$ and treated with 2,6-lutidine $(16 \mu \mathrm{mL}, 0.27 \mathrm{mmol})$ and TBSOTf ( $50 \mu \mathrm{mL}, 0.21 \mathrm{mmol})$ at $0{ }^{\circ} \mathrm{C}$. The reaction mixture was stirred for $1 \mathrm{~h}$ and quenched with saturated aqueous $\mathrm{NaHCO}_{3}(5 \mathrm{~mL})$ and extracted with EtOAc $(2 \times 10 \mathrm{~mL})$. The combined organic extracts were washed with saturated aqueous $\mathrm{CuSO}_{4}(2 \times 8 \mathrm{~mL})$, water $(2 \times 8 \mathrm{~mL})$, and brine $(10 \mathrm{~mL})$. After drying over anhydrous $\mathrm{Na}_{2} \mathrm{SO}_{4}$ and filtering, the solvent was evaporated under reduced pressure, and the residue was purified by column chromatography $\left(\mathrm{SiO}_{2}, 4 \% \mathrm{EtOAc} /\right.$ hexane), which afforded the target 3 as a colorless oil $(111 \mathrm{mg}, 69 \%) . R_{f}=0.4$ $\left(\mathrm{SiO}_{2}, 20 \%\right.$ EtOAc/hexane); $[\alpha]_{D}{ }^{30}-15.9$ (c 1.8, $\mathrm{CHCl}_{3}$ ).

IR (neat): $v_{\max }=2929,2856,1514,1464,1249,1106,835,773,702$ $\mathrm{cm}^{-1}$.

${ }^{1} \mathrm{H}$ NMR $\left(400 \mathrm{MHz}, \mathrm{CDCl}_{3}\right): \delta=7.70-7.64(\mathrm{~m}, 4 \mathrm{H}), 7.44(\mathrm{~m}, 6 \mathrm{H}), 7.25-$ $7.22(\mathrm{~m}, 2 \mathrm{H}), 6.91-6.85(\mathrm{~m}, 2 \mathrm{H}), 4.43(\mathrm{q}, J=11.7 \mathrm{~Hz}, 2 \mathrm{H}), 4.31(\mathrm{~m}, 1$ H), 4.07-3.88 (m, 2 H), 3.80 (s, 3 H), 3.68-3.47 (m, 6 H), 2.35 (dd, $J=$ $16.5,4.8 \mathrm{~Hz}, 1 \mathrm{H}), 2.59$ (dd, $J=16.5,3.6 \mathrm{~Hz}, 1 \mathrm{H}), 1.88-1.66(\mathrm{~m}, 3 \mathrm{H})$, 1.66-1.58 (m, $4 \mathrm{H}), 1.58-1.43$ ( $\mathrm{m}, 5 \mathrm{H}), 1.42(\mathrm{~s}, 3 \mathrm{H}), 1.41-1.37(\mathrm{~m}, 7$ H), $1.41(\mathrm{~s}, 3 \mathrm{H}), 1.37(\mathrm{~s}, 3 \mathrm{H}), 1.05(\mathrm{~s}, 9 \mathrm{H}), 0.89(\mathrm{~s}, 9 \mathrm{H}), 0.88(\mathrm{~s}, 18 \mathrm{H})$, $0.11(\mathrm{~s}, 3 \mathrm{H}), 0.09(\mathrm{~s}, 3 \mathrm{H}), 0.03(\mathrm{~s}, 12 \mathrm{H})$.

${ }^{13} \mathrm{C}$ NMR $\left(100 \mathrm{MHz}, \mathrm{CDCl}_{3}\right): \delta=159.4,135.8,134.4,131.7,130.8$, 130.8, 129.7, 129.4, 127.8, 114.0, 99.0, 84.2, 80.2, 72.9, 72.5, 72.5, $68.2,66.2,66.1,64.2,63.4,55.5,39.5,37.7,37.7,37.1,37.0,36.8,36.6$, $33.1,30.3,27.1,26.8,26.2,26.1,21.9,21.5,21.3,20.1,19.4,18.5,18.4$, $-4.1,-4.7$.

MS (ESI): $m / z=1162\left[\mathrm{M}+\mathrm{NH}_{4}\right]^{+}$.

HRMS: $m / z$ calcd for $\mathrm{C}_{66} \mathrm{H}_{116} \mathrm{O}_{8} \mathrm{Si}_{4} \mathrm{~N}\left[\mathrm{M}+\mathrm{NH}_{4}\right]^{+}:$1162.7773; found: 1162.7772

\section{Conflict of Interest}

The authors declare no conflict of interest.

\section{Funding Information}

N.S.K. thanks the University Grants Commission (UGC) New Delhi for a fellowship, and B.J.R. thanks the Science and Engineering Research Board (SERB) for a National Post Doctoral Fellowship (N-PDF).

\section{Acknowledgment}

We wish to thank the KIM department for providing the IICT ccommunication No. IICT/Pubs./2021/254.

\section{Supporting Information}

Supporting information for this article is available online at https://doi.org/10.1055/a-1647-7202.

\section{References}

(1) (a) Pallela, R.; Na-Young, Y.; Kim, S. K. Mar. Drugs 2010, 8, 1189. (b) Kuttruff, C. A.; Eastgate, M. D.; Baron, P. S. Nat. Prod. Rep. 2014, 31, 419. (c) Blunt, J. W.; Copp, B. R.; Keyzers, R. A.; Munro, M. H. G.; Prinsep, M. R. Nat. Prod. Rep. 2012, 29, 144.

(2) Shao, C.-L.; Linington, R. G.; Balunas, M. J.; Centeno, A.; Boudreau, P.; Zhang, C.; Engene, N.; Spadafora, C.; Mutka, T. S.; Kyle, D. E.; Gerwick, L.; Wang, C.-Y.; Gerwick, W. H. J. Org. Chem. 2015, 80, 7849.

(3) (a) Ghosh, S.; Kumar, S. U.; Shashidhar, J. J. Org. Chem. 2008, 73, 1582. (b) Ghosh, S.; Pradhan, T. K. J. Org. Chem. 2010, 75, 2107. (c) Athe, S.; Chandrasekhar, B.; Roy, S.; Pradhan, T. K.; Ghosh, S. J. Org. Chem. 2012, 77, 9840. (d) Reddy, K. M.; Yamini, V.; Singarapu, K. K.; Ghosh, S. Org. Lett. 2014, 16, 2658. (e) Chandrasekhar, B.; Athe, S.; Reddy, P. P.; Ghosh, S. Org. Biomol. Chem. 2015, 13, 115. (f) Rao, K. N.; Kanakaraju, M.; Kunwar, A. C.; Ghosh, S. Org. Lett. 2016, 18, 4092. (g) Athe, A.; Sharma, A.; Marumudi, K.; Ghosh, S. Org. Biomol. Chem. 2016, 14, 6769. (h) Sharma, A.; Athe, S.; Ghosh, S. ACS Omega 2018, 3, 16563.

(4) Quintard, A.; Sperandio, C.; Rodriguez, J. Org. Lett. 2018, 20, 5274.

(5) Schleicher, D. K.; Jamison, T. F. Beilstein J. Org. Chem. 2013, 9, 1533.

(6) (a) Chang, J.; Paquette, L. A. Org. Lett. 2002, 4, 253. (b) Paquette, L. A.; Chang, J.; Liu, Z. J. Org. Chem. 2004, 69, 6441.

(7) Sayini, R.; Srihari, P. Synthesis 2018, 50, 663.

(8) Nicolaou, K. C.; Daines, R. A.; Uenishi, J.; Li, W. S.; Papahatjis, P. D.; Chakraborty, T. K. J. Am. Chem. Soc. 1988, 110, 4672.

(9) Bujaranipalli, S.; Das, S. Tetrahedron: Asymmetry 2016, 27, 254.

(10) Cook, C.; Guinchard, X.; Liron, F.; Roulland, E. Org. Lett. 2010, 12, 744.

(11) Nilewski, C.; Deprez, N. R.; Fessard, T. C.; Li, D.; Geisser, R. W.; Carreira, E. M. Angew. Chem. Int. Ed. 2011, 50, 7940.

(12) Dess, D. B.; Martin, J. C. J. Am. Chem. Soc. 1991, 113, 7277.

(13) Matsumura, K.; Hashiguchi, S.; Ikariya, T.; Noyori, R. J. Am. Chem. Soc. 1997, 119, 8738 . 